\title{
High Photoconductivity in Heavily Doped GaAs/AlAs Superlattices with Electric Domains
}

\author{
$\underline{\text { S. K. Paprotskiy }}^{1}$, I. V. Altukhov ${ }^{1}$, M. S. Kagan ${ }^{1}$, N. A. Khvalkovskiy ${ }^{1}$, \\ I. S. Vasil'evskii ${ }^{2}$, A. N. Vinichenko ${ }^{2}$ \\ ${ }^{1}$ V.A. Kotel'nikov Institute of Radio Engineering and Electronics, Russian Ac. Sci., Moscow, Russia, \\ s.paprotskiy@gmail.com \\ ${ }^{2}$ National Research Nuclear University MEPhI, Moscow, Russia
}

In last three decades, the electron transport in semiconductor superlattices (SLs) has been studied in detail $[1,2]$, mainly in connection with the predicted amplification of Bloch waves, which can make it possible to create tunable sources of $\mathrm{THz}$ radiation. The main hindrance to the Bloch wave amplification is the formation of electrical domains owing to the static negative differential conductivity (NDC) arising in superlattices at resonant tunneling between confined states in neighboring quantum wells. Many works were devoted also to studies of domain properties (see, e.g., [2] and references therein). Most of them were performed with weakly doped SLs at low temperatures under strong illumination [2-7]. The change in free carrier concentration under illumination gives rise to different domain regimes. It has been shown, in particular, that transition from travelling to static domain regime occurs at sufficiently high illumination intensity. In this work studies of effect of interband illumination on tunneling current in highly doped short-period superlattices at the domain formation are presented.

We used the MBE grown GaAs/AlAs SLs consisted of 100 periods of $4 \mathrm{~nm} \mathrm{GaAs} / 2 \mathrm{~nm} \mathrm{AlAs}$ between heavily doped $\left(\sim 10^{19} \mathrm{~cm}^{-3}\right) \mathrm{n}^{+}$cap layer and $\mathrm{n}^{+}$ substrate. GaAs quantum wells (QWs) were Si doped with concentration of $2 * 10^{17} \mathrm{~cm}^{-3}$. A cross-section of the structure is shown in Fig. 1.

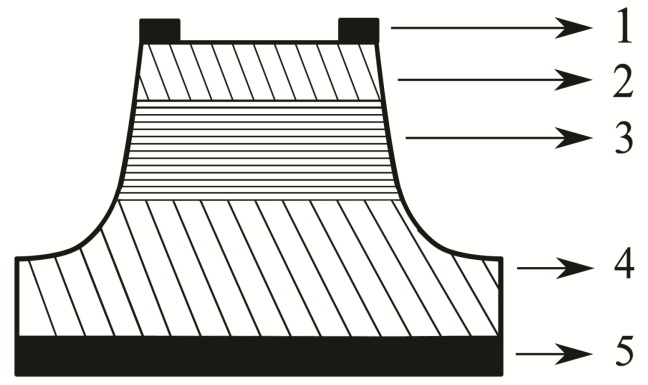

Fig. 1. Scheme of the structure: $(1,5)$ top and bottom metal contacts, (2) top contact $n^{+}$layer, (3) superlattice, and (4) heavily doped substrate.

Triangular voltage pulses with sweep-up times of 0.2 to $10 \mu \mathrm{s}$ and sweep-down times up to $100 \mu \mathrm{s}$ were used to record I-V curves. Measurements were performed at room temperature.

A sharp decrease of current at some threshold voltage (Fig. 2) indicates the moving domain formation. The current rise in the mean is an evidence of triangular form of the domain [2, 9]. The series of maxima in current-voltage characteristics is attributed to the optical phonon assisted tunneling between quantum wells inside the triangular domain.

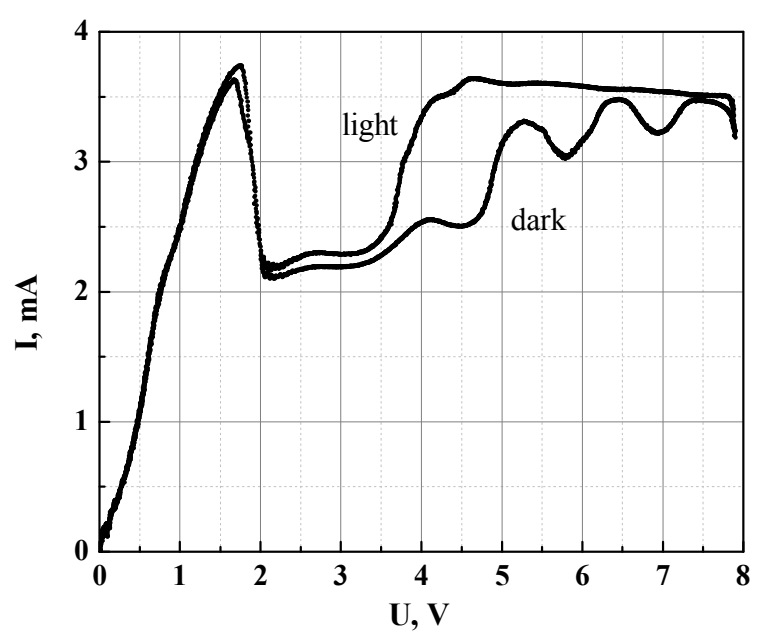

Fig. 2. Current-voltage characteristics of the GaAs/AlAs SL in the dark (lower curve) and under band-to-band illumination (upper curve).

A strong effect of rather weak illumination (with a LED or a filament bulb) on a tunneling current in GaAs/AlAs SLs with relatively high doping level was found under conditions of electric domains formation. Fig. 2 shows the current-voltage characteristics with and without illumination. The photocurrent at the domain regime could reach $50 \%$ of the dark current, while it was several orders of magnitude less at voltages below the threshold one (before the domain formation) as the free carrier concentration excited with light was considerably less than $\mathrm{Si}$ donor concentration. The measured photocurrent spectrum (Fig. 3) points to the band-to-band excitation. The spectral long-wavelength cutoff allows us to determine the GaAs QW gap and the energy of first size quantization level. These energies coincide with known values for GaAs QW.

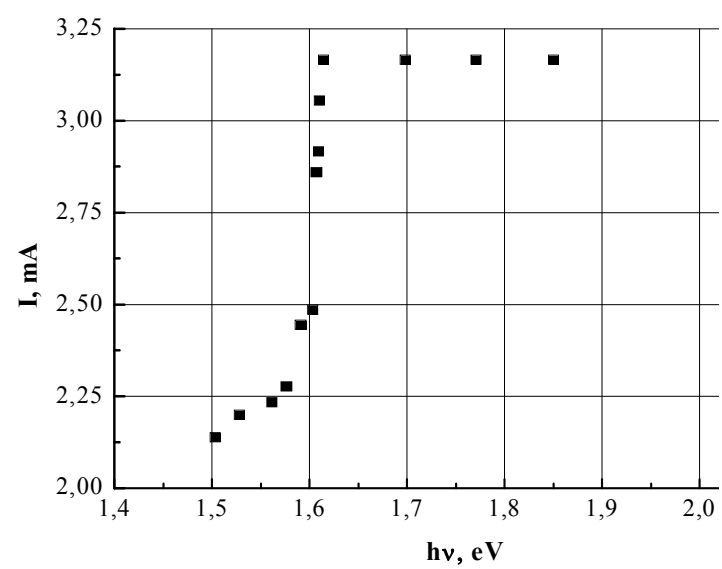

Fig. 3. Photocurrent spectrum 
The large photocurrent at the domain formation is referred to the triangular form of high-field domain with wide region of donor depopulation. The free carrier concentration in this region is very small and additional concentration of electron-hole pairs created by interband illumination exceeds significantly a stationary concentration inside the domain, which results in a change of space charge at domain boundaries. This leads to a change of domain movement regime.

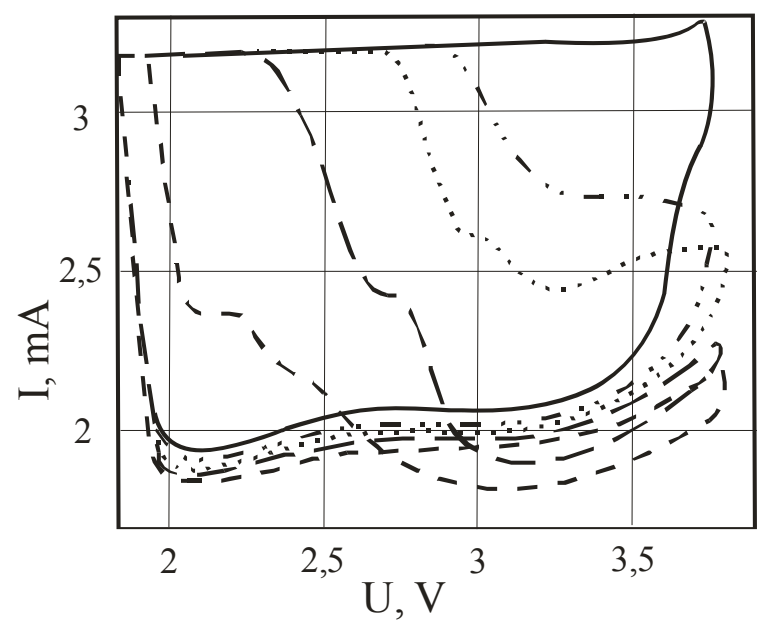

Fig. 4. Parts of current-voltage characteristics of the $\mathrm{GaAs} / \mathrm{AlAs} \mathrm{SL}$ in an intermediate range of domain existence at different light intensities.

The sufficiently intense illumination could initiate the travelling-to-static domain transition, which becomes apparent in current saturation regions in I-V curves (Fig. 2). Shown in Fig. 4 are parts of current-voltage characteristics corresponding to an intermediate voltage range of domain existence for different illumination intensities. I-V curves were measured at forward and backward bias sweep. The current hysteresis is connected with a large asymmetry of transition times from moving to static field domain and back at voltage sweep-up and sweep-down, respec- tively [10]. In this voltage range (compare with Fig. 2 ), the current saturation was observed only at backward voltage sweep.

The work was supported by RFBR grants 16-2903135 and 16-29-09626

\section{References}

1. Wacker, A. Semiconductor superlattices: a model system for nonlinear transport // Physics Reports, 2002. V. 357, P. 1

2. Bonilla, L. L., Grahn H. T. Non-linear dynamics of semiconductor superlattices // Rep. Prog. Phys. 2005. V. 68, No. 3, P. 577

3. Sun B. Q., Jiang D. S. and Wang X. J. Current oscillations and stable electric field domains in doped GaAs/AlAs superlattices // Semicond. Sci. Technol. 1997, V. 12, No 4, P. 401

4. Tomlinson A. M., Fox A. M., Cunningham J. E. and Jan $W$. $Y$. Photocurrent self-oscillations in a spatially direct GaAs/AlGaAs superlattice // Appl. Phys. Lett. 1999, V. 75, P. 2067

5. Kwok S. H., Grahn H. T. et al. Nonresonant carrier transport through high-field domains in semiconductor superlattices // Phys. Rev. B. 1995. V. 51. P. 9943

6. Kwok S. H., Norris T. B., Bonilla, L. L., et al. Domain-wall kinetics and tunneling-induced instabilities in superlattices // Phys. Rev. B 1995. V. 51. P. 10171

7. Grahn H. T., Schneider H., v. Klitzing K. Optical studies of electric field domains in $\mathrm{GaAs} / \mathrm{Al}_{\mathrm{x}} \mathrm{Ga}_{1-\mathrm{x}}$ As superlattices // Phys. Rev. B 1990. V. 41, P. 2890

8. Ohtani N., Egami N., Grahn H. T., Ploog K. H., and Bonilla L. L. Transition between static and dynamic electric-field domain formation in weakly coupled GaAs/AlAs superlattices // Phys. Rev. B 1998. V. 58, P. R7528(R)

9. Suris R. A. Inhomogeneous structures in semiconductors with superlattices // Sov. Phys. Semicond. 1973. V. 7, No. 8, P. 1035

10. Altukhov I. V., Kagan M. S., Paprotskiy S. K., Khvalkovskiy N. A., Vasil'evskii I. S., Vinichenko A. N. Electronic Tunneling and Electric Domains in GaAs/AlAs Superlattices at Room Temperature, this issue. 